\title{
Morcegos (Mammalia: Chiroptera) da região do Médio Rio Teles Pires, Sul da Amazônia, Brasil
}

João M. D. MIRANDA*1,2, Luciana ZAGO3, Fernando CARVALHO², Marcelo B. G. RUBIO², Itiberê P. BERNARDI ${ }^{3}$

\footnotetext{
1 Universidade Estadual do Centro-Oeste do Paraná, Departamento de Biologia. Rua: Simeão Camargo Varela de Sá, 03, Vila Carli. Guarapuava, Brasil. CEP: 85.040-080.

2 Universidade Federal do Paraná, campus Centro Politécnico, Setor de Ciências Biológicas, Programa de Pós-Graduação em Zoologia, Rua: Cel. Francisco H. dos Santos, 210, Jardim das Américas, Curitiba, Brasil. CEP: 81.531-970.

${ }^{3}$ Universidade Federal do Paraná, campus Centro Politécnico, Setor de Ciências Biológicas, Programa de Pós-Graduação em Ecologia e Conservação ,Rua: Cel. Francisco H. dos Santos, 210, Jardim das Américas, Curitiba, Brasil. CEP: 81.5131-970.

* Autor correspondente: guaribajoao@yahoo.com.br
}

\section{RESUMO}

A Amazônia é a maior floresta tropical do mundo e pelo menos 147 espécies de morcegos ocorrem neste ambiente. A despeito desta grande riqueza, a diversidade de morcegos da Amazônia é pobremente conhecida e existem grandes lacunas neste conhecimento. O objetivo do presente trabalho foi descrever a assembleia de morcegos ocorrentes na região do Médio Teles Pires (MTP), no sul da Amazônia. Além disso, avaliou-se a similaridade dessa assembleia em relação a 14 assembleias estudadas em outras localidades amazônicas e avaliou-se a correlação entre as similaridades destas localidades e suas distâncias. Trinta e três espécies de morcegos foram registradas, representando $71 \%$ das espécies estimadas (Jackknife2). As três espécies com maior abundância relativa foram: Carollia perspicillata, Pteronotus parnellii e Phyllostomus hastatus que somadas contam com mais de $50 \%$ das capturas. O grupo funcional dos frugívoros obteve o maior número de espécies capturadas. Foi encontrada uma correlação negativa entre as distâncias e as similaridades das assembleias de morcegos amazônicos $(\mathrm{r}=-0,22 ; \mathrm{p}=0,014)$. A distância geográfica pode explicar apenas $6 \%$ da similaridade entre as assembleias analisadas, ainda assim, as similaridades destas assembleias permitem que as mesmas sejam agrupadas por suas distâncias geográficas. Além disso, a fauna de morcegos do MTP é diferenciada de outras áreas da Amazônia o que lhe confere um papel especial na conservação dos morcegos amazônicos. PALAVRAS-Chave: Chiroptera, Inventário de fauna, Assembleias, Biodiversidade.

\section{Bats (Mammalia: Chiroptera) from the Middle Teles Pires River region, Southern Amazonia, Brazil}

\begin{abstract}
Amazonia is the largest tropical rainforest in the world and at least 147 bat species occur in this environment. Despite its richness, the diversity of bat fauna is poorly known for this region and there are large gaps in its knowledge. This study aims to describe the bat assemblage of the Middle Teles Pires River (MTP) region, southern Amazonia. Furthermore, we evaluated the similarity of this assemblage in relation to 14 assemblages studied in other Amazonian localities, and correlations were found between the similarity values among the sites and their geographic distances. Thirty-three bat species were recorded, accounting for $71 \%$ of the estimated species richness (Jackknife2). The three species that had the higher relative abundance values were Carollia perspicillata, Pteronotus parnellii and Phyllostomus hastatus, which together accounted for more than 50\% of the captures. The frugivore functional group had the highest number of recorded species. A negative correlation was found between the geographic distances and the similarities among the Amazonian bat assemblages $(r=-0.22 ; p=0.014)$. Geographic distance could explain only $6 \%$ of the similarities among the analyzed assemblages, even though, the similarity found allowed them to be grouped by their distances. Besides, the MTP bat fauna is different from other Amazonian areas, which gives it a special role in the Amazonian bat conservation.
\end{abstract}

KEYWORDS: Chiroptera, Fauna survey, Assemblage, Biodiversity. 


\section{INTRODUÇÃO}

A Amazônia é a maior floresta tropical do mundo e abriga uma grande diversidade biológica (Heyer et al. 1999; Magurran 2011). A origem dessa diversidade biológica é de interesse dos cientistas desde a época de Darwin e Wallace, no século XIX (Bush e Oliveira 2006). Várias hipóteses tentam explicar os padrôes de biodiversidade amazônica como por exemplo: a hipótese dos refúgios de fauna (Haffer 1969; Vanzolini 1992), a hipótese de que existem maiores áreas tropicais que sub-tropicais ou temperadas (Rosenzweig e Sandlin 1997) e a teoria da barreira fluvial (Hershkovitz 1977; van Roosmalen et al. 2002). De maneira geral essas hipóteses tratam de barreiras geográficas impostas aos organismos modelos estudados (aves florestais, lagartos e primatas) (Bush e Oliveira 2006). Uma das principais críticas às várias teorias é que cada grupo animal possui sua própria história evolutiva, necessidades ambientais e capacidade de dispersão (Haffer e Prance 2002; Bush e Oliveira 2006). Os morcegos são animais voadores e possuem altas capacidades de dispersão e amplas distribuiçôes geográficas (Kunz e Pierson 1994; Gardner 2008), e podem responder de maneiras diferentes a uma mesma barreira.

Os morcegos apresentam as maiores riquezas entre as comunidades de mamíferos da Amazônia servindo como bons modelos para estudos sobre a diversidade e a biogeografia (Voss e Emmons 1996; Moratelli et al. 2010), bem como bons indicadores de processos ecológicos (Passos et al. 2003). Por outro lado, o conhecimento sobre a biodiversidade de morcegos da Amazônia é bastante assimétrico com boas amostragens ao longo da calha do rio Amazônas e do rio Tapajós e também no Escudo Guiano (e.g.Simmons e Voss 1998; Bernard 2002; Sampaio et al. 2003), porém as regiōes Oeste e Sul permanecem virtualmente desconhecidas (Bernard et al. 2010; Martins et al. 2011). Assim, o presente estudo teve por objetivo: (I) descrever a estrutura de uma assembleia de morcegos em uma região mal amostrada ao sul da Amazônia; (II) avaliar se a fauna de morcegos da Amazônia apresenta alguma regionalização e se esta pode ser explicada pela distância geográfica entre as áreas amostradas.

\section{MATERIAL E MÉTODOS}

A área de estudo compreende extensas áreas de florestas às margens do Médio Rio Teles Pires (MTP) entre os municípios de Nova Canaã do Norte e Itaúba, no norte do estado do Mato Grosso (10 58' 04” S; 55 45’ 23” W). A regiāo do MTP está localizada próxima aos limites dos biomas Amazônia e Cerrado (Figura 1). A área é formada por floresta tropical madura com lianas formando um dossel descontínuo, estando este compreendido entre 20

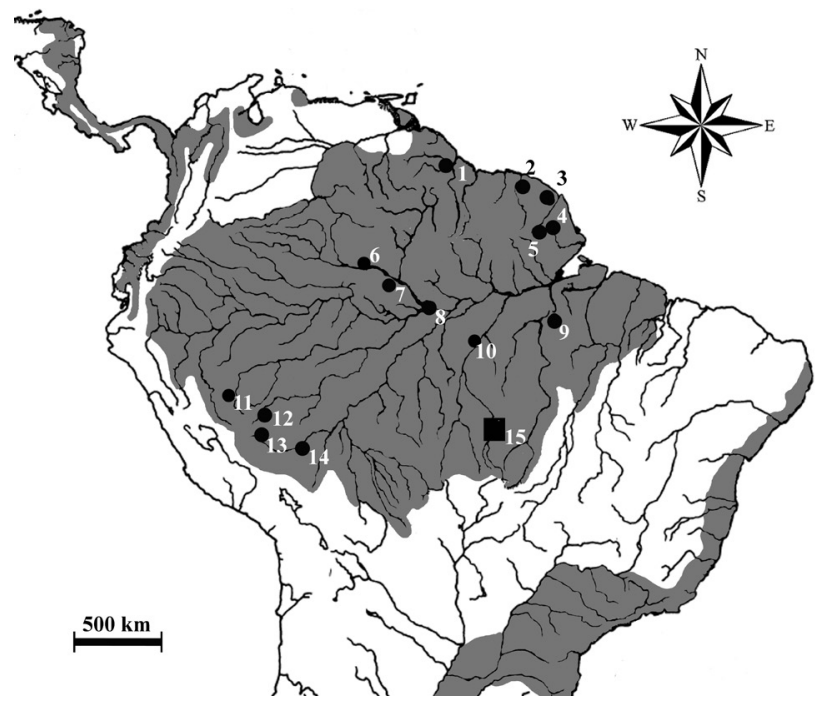

Figura 1. Mapa mostrando a área de estudo e outras localidades citadas no texto. Áreas em cinza indicam florestas úmidas das Américas. (1) Iwokrama Forest, Guiana (IWO); (2) Paracou, Guiana Francesa (PAR); (3) Baixo Arataye, Guiana Francesa (ARA); (4) Floresta Nacional do Amapá, Amapá, Brasil (FLAM); (5) Parque Nacional das Montanhas do Tumucumaque, Amapá, Brasil (PNMT); (6) Barcelos e Santa Isabel do Rio Negro, Amazonas (BAR); (7) Parque Nacional do Jaú, Amazonas, Brasil (PNJ); (8) Manaus, Amazonas, Brasil (MAN); (9) Baixo Xingu, Pará (XIN); (10) Parque Nacional da Amazônia, Pará/Amazonas, Brasil (PNA); (11) Parque Nacional da Serra do Divisor, Acre, Brasil (PNSD); (12) Aldeia Indígena Cashinahua de Balta, Ucayali, Peru (BAL); (13) Estación Biológica Cosha Cashu, Rio Manu, Madre de Dios, Peru (MNU); (14) Reserva Cuzco Amazónico, Madre de Dios, Peru (CUZ) e (15) Médio Teles Pires, Mato Grosso, Brasil (MTP).

e 30 metros de altura, com árvores emergentes alcançando até $40 \mathrm{~m}$. O dossel é relativamente denso e a floresta bem estratificada (Gualda-Barros et al. 2012). O clima é equatorial quente e semi-úmido com pelo menos três meses de estação seca e temperaturas mensais médias sempre acima dos $18^{\circ} \mathrm{C}$ (IBGE 2002).

$\mathrm{Na}$ tentativa de melhor amostrar a diversidade de ambientes no MTP as amostragens foram realizadas em três fitofisionomias, chamadas daqui em diante de sítios amostrais: (1) Floresta Ombrófila Densa Aluvial (FAlu) à margem esquerda do rio Teles Pires e a $250 \mathrm{~m}$ de altitude; (2) Floresta Ombrófila Densa Submontana (FSub) a 2,5 km da margem direita do rio Teles Pires e a $350 \mathrm{~m}$ de altitude e (3) Floresta Ombrófila Densa Aluvial Secundária (FSec) a $1 \mathrm{~km}$ da margem direita do rio Teles Pires e a $245 \mathrm{~m}$ de altitude. A menor distância entre as áreas foi entre a FSub e a FAlu $(2,65 \mathrm{~km})$, sendo seguida pela FSub e a FSec $(2,75$ $\mathrm{km})$ e as mais distantes foram a FSec e a FAlu $(5,02 \mathrm{~km})$.

Entre fevereiro de 2011 e abril de 2012, foram realizadas seis campanhas de campo com duraçáo de seis noites consecutivas de amostragem sendo duas noites em cada um dos três sítios amostrais, o que resultou em 36 
noites (12 noites em cada sítio amostral). As noites de amostragem foram sempre durante a fase lunar minguante ou nova. Foram realizadas três idas a campo na estação seca e outras três na estação chuvosa. Em cada noite de amostragem foram armadas 12 redes de neblina $(6 \times 3 \mathrm{~m})$ que permaneceram abertas por seis horas após o pôr-do-sol, sendo revisadas em intervalos de $30 \mathrm{~min}$. As redes foram armadas no nível do sub-bosque em trilhas e clareiras préexistentes e também próximas a rios. Esse esforço amostral totalizou $46.656 \mathrm{~m}^{2} \mathrm{hr}^{-1}$ (segundo Bianconi e Straube 2002), sendo esse distribuído igualitariamente entre os três sítios amostrais. Os indivíduos capturados foram triados e identificados de acordo com a literatura disponível (Lim e Engstron 2001; Reis et al. 2007; Gardner 2008). Os primeiros exemplares de cada espécie foram coletados e tombados na Coleçáo Científica de Mastozoologia da Universidade Federal do Paraná como material testemunho. Os demais espécimes capturados foram marcados com anilhas metálicas numeradas e coloridas e soltos no mesmo local da captura. As capturas, o manejo e os procedimentos dos animais foram feitas sob a licença do SISBIO/IBAMA No 02013004996/10-08 e seguiram as indicaçóes da American Society of Mammalogists (Sikes et al. 2011).

As diversidades específicas (Diversidade de ShannonWiener) de morcegos foram comparadas entre os três sítios amostrais (FAlu, FSub e FSec) pelo teste $t$ de Shannon. Uma vez que não houve diferença significativa $(p>0,05)$ entre qualquer um dos três sítios amostrais esses foram tratados nas demais análises de forma conjunta, compondo uma única assembleia de morcegos do MTP.

A riqueza estimada de morcegos foi calculada através do estimador náo paramétrico Jackkinfe de segunda ordem (Jackknife2). Essa estimativa foi obtida por meio de 1.000 re-amostragens (bootstraping) dos dados originais gerando medidas de desvio padrão. A avaliação do esforço amostral foi feita pela análise de complementaridade no pior cenário, dado pela porcentagem das espécies registradas em relação ao número médio de espécies estimada mais o desvio padrão obtido pelas re-amostragens.

A abundância relativa foi calculada pela porcentagem que cada espécie representa no total de capturas. A frequência relativa das espécies foi calculada dividindo-se o número de campanhas de campo em que cada espécie foi registrada pelo número total de fases de campo (seis). A partir de suas frequencias relativas cada espécie foi classificada quanto à sua constância como: Comum quando a freqüência relativa foi $\geq 50 \%$; Intermediária quando a freqüência relativa foi entre 25 e $50 \%$ ou Rara quando a freqüência relativa foi < 25\% (como em Bianconi et al. 2004; Carvalho et al. 2013).

Cada espécie registrada foi categorizada em um dos seguintes grupos funcionais como em Simmons e Voss (1998): (1) Insetívoros aéreos (todos os não Phyllostomidae), (2) Catadores animalívoros (todos os Phyllostominae, exceto
Phylloderma stenops, Phyllostomus hastatus e P. discolor), (3) Frugívoros (todos os Carolliinae e Stenodermatinae), (4) Nectarívoros (todos os Glossophaginae), (5) Hematófagos (todos os Desmodontinae) e (6) Onívoros (P. stenops, $P$. hastatus e $P$. discolor).

Para as análises de Agrupamento (Cluster) e de relação (Correlação e Regressão) entre similaridade das assembleias de morcegos e distância das áreas onde essas assembleias de morcegos foram estudadas (ver abaixo), foram utilizadas as informaçôes de riqueza de 15 assembleias da Amazônia, sendo estas as localidades: (1) Iwokrama Forest, Guiana (IWO) (Lim e Engstron 2001); (2) Paracou, Guiana Francesa (PAR) (Simmons e Voss 1998); (3) Baixo Arataye, Guiana Francesa (ARA) (Voss e Emmons 1996); (4) Floresta Nacional do Amapá, Amapá, Brasil (FLAM) (Martins et al. 2006); (5) Parque Nacional das Montanhas do Tumucumaque, Amapá, Brasil (PNMT) (Martins et al. 2006); (6) Barcelos e Santa Isabel do Rio Negro, Amazonas (BAR) (Moratelli et al. 2010); (7) Parque Nacional do Jaú, Amazonas, Brasil (PNJ) (Barnett et al. 2006); (8) Manaus, Amazonas, Brasil (MAN) (Reis e Peracchi 1987); (9) Baixo Xingu, Pará (XIN) (Voss e Emmons 1996); (10) Parque Nacional da Amazônia, Pará/Amazonas, Brasil (PNA) (Bernard 2001); (11) Parque Nacional da Serra do Divisor, Acre, Brasil (PNSD) (Nogueira et al. 1999); (12) Aldeia Indígena Cashinahua de Balta, Ucayali, Peru (BAL) (Voss e Emmons 1996); (13) Estación Biológica Cosha Cashu, Rio Manu, Madre de Dios, Peru (MNU) (Voss e Emmons 1996); (14) Reserva Cuzco Amazónico, Madre de Dios, Peru (CUZ) (Voss e Emmons 1996) e (15) Médio Teles Pires, Mato Grosso, Brasil (MTP) (presente trabalho) (Figura 1). No sentido de padronizar as assembleias amostradas, uma vez que alguns dos trabalhos supracitados utilizaram de redes armadas no dossel da floresta (Simmons e Voss 1998; Bernard 2001; Lim e Engstron 2001; Barnett et al. 2006), nessas três análises (correlação, regressão e agrupamento) foram consideradas apenas as espécies capturadas no subbosque da floresta (como em Moratelli et al. 2010).

Foi realizada uma Análise de Agrupamento (Cluster) (através do algoritmo UPGMA - Unweighted pair-group average) com o Coeficiente de Similaridade de Jaccard para comparar as 15 assembleias de morcegos utilizadas no presente estudo. Para essa Análise de Agrupamento foi utilizada uma matriz binária com a presença ou ausência de espécies por área estudada (Anexo 1). Na Análise de Agrupamento foram feitas 100 re-amostragens (Bootstraping) no sentido de verificar quanto cada ramo do dendrograma é suportado. Para testar se há correlação entre similaridade (Similaridade de Jaccard) e distância das assembleias foram obtidas as distâncias (em km) em linha reta entre cada par de localidades amostradas com a ferramenta de régua no software Google Maps. Foram obtidos também o valor do Coeficiente de Similaridade de 
Jaccard para riqueza de espécies de morcegos entre cada par das 15 localidades amostradas. Foi realizado o teste de Correlaçáo de Pearson entre as distâncias (em km) de cada par de localidades amostradas e o valor de Coeficiente de Similaridade de Jaccard de cada mesmo par de localidades. Quando a correlaçáo foi significativa (análise anterior) procedeu-se uma análise de Regressão (através do algoritmo RMA - Reduced Major Axis) afim de se verificar quanto da similaridade da fauna pode ser explicada pela distância entre as áreas. Todas as análises foram feitas no software $\mathrm{PAST}^{\circ}$ versão 2.14 (Hammer 2012) adotando-se o nível de significância $\mathrm{p}<0,05$.

\section{RESULTADOS}

Foram obtidas 337 capturas (excluindo-se 12 recapturas) que representaram 33 espécies, 25 gêneros e 6 famílias (Tabela 1). A riqueza total do MTP foi estimada em 36,6 \pm 9,9 espécies pelo estimador Jackknife2. Isso indica que o esforço realizado amostrou pelo menos $71 \%$ da riqueza estimada (no pior cenário).

As espécies com maior abundância relativa na amostragem geral foram: Carollia perspicillata (25,8\%), Pteronotus parnellii $(13,4 \%)$ e Phyllostomus hastatus $(11,3 \%)$, as demais espécies foram representadas por menos que $10 \%$ dos indivíduos capturados. Doze espécies foram consideradas Comuns, seis espécies Intermediárias e 16 espécies Raras na região do MTP. O grupo funcional com o maior número de espécies foi de morcegos Frugívoros contando com dez espécies, seguido pelos Insetívoros aéreos e os Catadores animalívoros com oito espécies cada. Os morcegos Nectarívoros e Onívoros foram representados por apenas três espécies cada, enquanto os Hematófagos foram representados por apenas uma espécie (Tabela 1).

A Análise de Agrupamento mostrou os seguintes agrupamentos geográficos e bem suportados: (1) as assembleias do Escudo Guiano; (2) as assembleias da Amazônia Ocidental; (3) as assembleias da Amazônia Central e (4) as assembleias do estado do Amapá (PNMT e FLAM) (Figura 2). A comunidade de morcegos do MTP foi mais similar às registradas no FLAM $(44,7 \%)$, no PNMT (44\%), no XIN $(42,8 \%)$ e no ARA $(42,4 \%)$ (Tabela 2). A correlaçáo entre a similaridade da fauna de morcegos e as respectivas distâncias entre áreas foi corroborada para as assembleias de morcegos da Amazônia, sendo encontrada uma correlação negativa $(r=-0,22 ; p=$ $0,014)$. A similaridade da fauna de morcegos das 15 áreas da Amazônia pôde ser explicada em apenas $6 \%$ pelas distâncias entre áreas $\left(\mathrm{r}^{2}=0,0622 ; \mathrm{F}=-2,48 ; \mathrm{p}=0,0133\right)$ (Figura 3).
Tabela 1. Espécies registradas na região do Médio Teles Pires, Mato Grosso seguidas de seus respectivos Grupos Funcionais (= GF), Abundâncias (= $A B)$, Abundâncias Relativas (=AR) e Constância (= CON) das espécies.

\begin{tabular}{|c|c|c|c|c|}
\hline Taxa (Espécie, Família, Subfamília) & GF & $A B$ & AR $(\%)$ & $\mathrm{CON}$ \\
\hline \multicolumn{5}{|l|}{ Família Emballonuridae } \\
\hline $\begin{array}{l}\text { Rhynchonycteris naso } \\
\text { (Wied-Neuwied, 1820) }\end{array}$ & $\mathrm{IA}$ & 3 & 0,9 & Intermediária \\
\hline \multicolumn{5}{|l|}{ Família Phyllostomidae } \\
\hline \multicolumn{5}{|l|}{ Subfamília Phyllostominae } \\
\hline Chrotopterus auritus (Peters, 1856) & $\mathrm{CA}$ & 1 & 0,3 & Rara \\
\hline $\begin{array}{l}\text { Glyphonycteris sylvestris } \\
\text { Thomas, } 1896\end{array}$ & $\mathrm{CA}$ & 1 & 0,3 & Rara \\
\hline $\begin{array}{l}\text { Lampronycteris brachyotis } \\
\text { (Dobson, 1879) }\end{array}$ & $\mathrm{CA}$ & 1 & 0,3 & Rara \\
\hline Lophostoma brasiliense Peters, 1866 & $\mathrm{CA}$ & 1 & 0,3 & Rara \\
\hline Phylloderma stenops Peters, 1865 & ONI & 1 & 0,3 & Rara \\
\hline Phyllostomus discolor Wagner, 1843 & ONI & 5 & 1,5 & Comum \\
\hline $\begin{array}{l}\text { Phyllostomus elongatus } \\
\text { (É. Geoffroy, 1810) }\end{array}$ & $\mathrm{CA}$ & 9 & 2,7 & Comum \\
\hline Phyllostomus hastatus (Pallas, 1767) & ONI & 38 & 11,3 & Comum \\
\hline $\begin{array}{l}\text { Phyllostomus latifolius } \\
\text { (Thomas, 1901) }\end{array}$ & $\mathrm{CA}$ & 2 & 0,6 & Intermediária \\
\hline Trachops cirrhosus (Spix, 1823) & $\mathrm{CA}$ & 2 & 0,6 & Intermediária \\
\hline Trinycteris nicefori (Sanborn, 1949) & $\mathrm{CA}$ & 1 & 0,3 & Rara \\
\hline \multicolumn{5}{|l|}{ Subfamília Glossophaginae } \\
\hline Glossophaga soricina (Pallas, 1766) & NEC & 1 & 0,3 & Rara \\
\hline Lionycteris spurrelli Thomas, 1913 & NEC & 2 & 0,6 & Rara \\
\hline Lonchophylla thomasi J.A. Allen, 1904 & NEC & 1 & 0,3 & Rara \\
\hline \multicolumn{5}{|l|}{ Subfamília Desmodontinae } \\
\hline $\begin{array}{l}\text { Desmodus rotundus } \\
\text { (É. Geoffroy, 1810) }\end{array}$ & HEM & 2 & 0,6 & Rara \\
\hline \multicolumn{5}{|l|}{ Subfamília Carolliinae } \\
\hline Carollia perspicillata (Linnaeus, 1758) & FRU & 87 & 25,8 & Comum \\
\hline Rhinophylla pumilio Peters, 1865 & FRU & 26 & 7,7 & Comum \\
\hline \multicolumn{5}{|l|}{ Subfamília Stenodermatinae } \\
\hline Artibeus (A.) lituratus (Olfers, 1818) & FRU & 15 & 4,5 & Comum \\
\hline Artibeus (A.) obscurus (Schinz, 1821) & FRU & 30 & 8,9 & Comum \\
\hline Artibeus (A.) planirostris Spix, 1823 & FRU & 22 & 6,5 & Comum \\
\hline Artibeus (D.) gnomus (Handley, 1987) & FRU & 11 & 3,3 & Comum \\
\hline $\begin{array}{l}\text { Mesophylla macconnelli } \\
\text { Thomas, } 1901\end{array}$ & FRU & 1 & 0,3 & Rara \\
\hline $\begin{array}{l}\text { Platyrrhinus fusciventris Velazco, } \\
\text { Gardner \& Patterson, } 2010\end{array}$ & FRU & 2 & 0,6 & Intermediária \\
\hline Uroderma bilobatum Peters, 1866 & FRU & 12 & 3,6 & Comum \\
\hline Vampyressa thyone Thomas, 1809 & FRU & 3 & 0,9 & Intermediária \\
\hline \multicolumn{5}{|l|}{ Família Mormoopidae } \\
\hline Pteronotus parnellii (Gray, 1843) & IA & 45 & 13,4 & Comum \\
\hline $\begin{array}{l}\text { Pteronotus personatus } \\
\text { (Wagner, 1843) }\end{array}$ & $\mathrm{IA}$ & 1 & 0,3 & Rara \\
\hline \multicolumn{5}{|l|}{ Família Noctilionidae } \\
\hline Noctilio albiventris Desmarest, 1818 & IA & 2 & 0,6 & Rara \\
\hline \multicolumn{5}{|l|}{ Família Molossidae } \\
\hline Molossus molossus (Pallas, 1766) & IA & 1 & 0,3 & Rara \\
\hline Molossus rufus É. Geoffroy, 1805 & IA & 2 & 0,6 & Rara \\
\hline \multicolumn{5}{|l|}{ Família Vespertilionidae } \\
\hline Myotis nigricans (Schinz, 1821) & $\mathrm{IA}$ & 4 & 1,2 & Intermediária \\
\hline Myotis riparius Handley, 1960 & IA & 2 & 0,6 & Rara \\
\hline
\end{tabular}




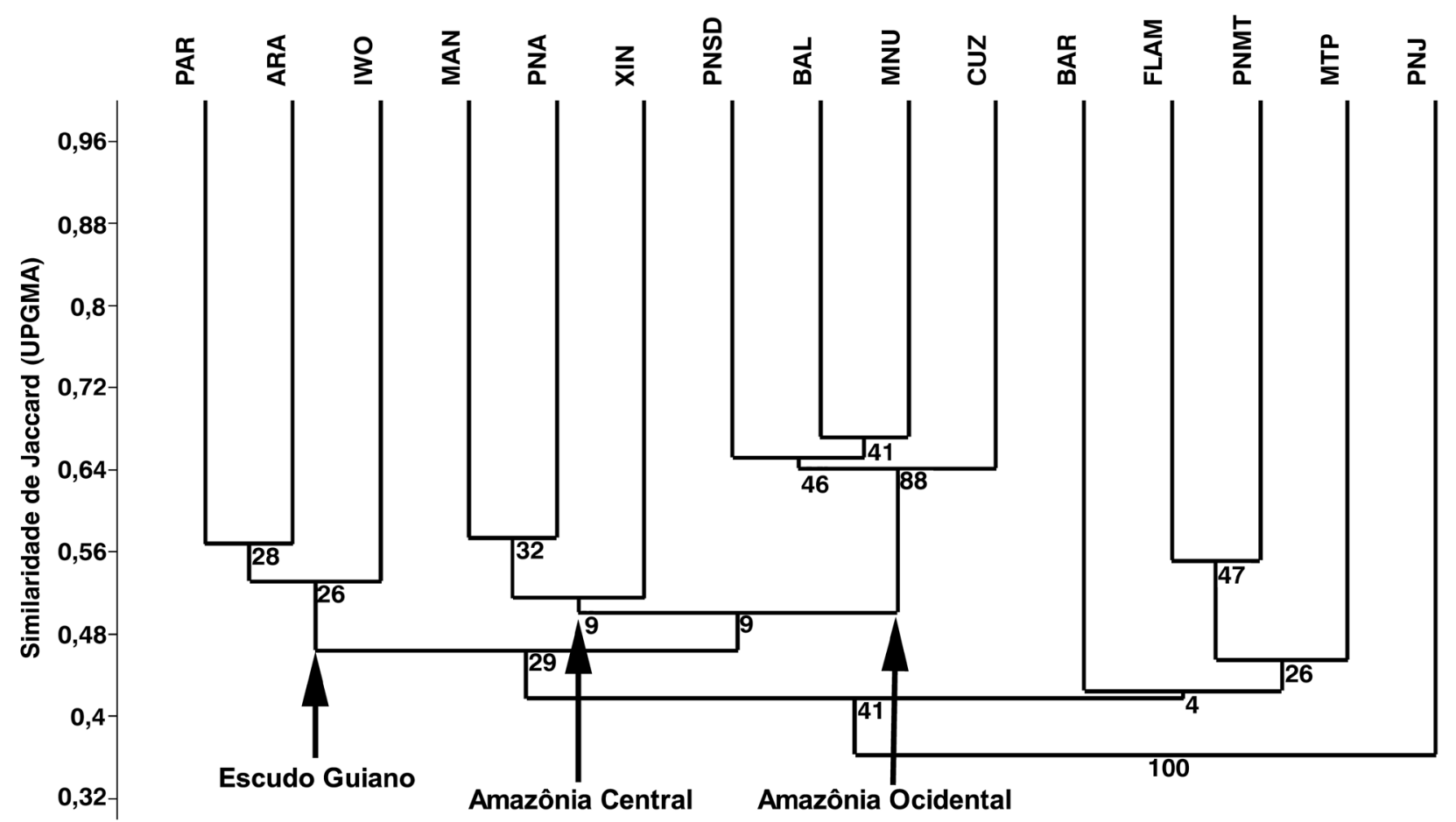

Figura 2. Análise de Agrupamento (UPGMA) através do Índice de Similaridade de Jaccard entre as seguintes assembleias de morcegos da Amazônia: IWO = Iwokrama Forest, Guiana; PAR = Paracou, Guiana Francesa; ARA = Baixo Arataye, Guiana Francesa; FLAM = Floresta Nacional do Amapá, Amapá, Brasil; PNMT = Parque Nacional das Montanhs do Tumucumaque, Amapá, Brasil; BAR = Barcelos e Santa Isabel do Rio Negro; PNJ = Parque Nacional do Jaú, Amazonas, Brasil; MAN = Manaus, Amazonas, Brasil; XIN = Baixo Xingú, Pará, Brasil; PNA = Parque Nacional da Amazônia, Amazonas/Pará, Brasil; PNSD $=$ Parque Nacional da Serra do Divisor, Acre, Brasil; BAL = Balta, Ucayali, Peru; MNU = Parque Nacional de Manu, Madre de Dios, Peru; CUZ = Reserva Cuzco Amazônico, Madre de Dios, Peru e MTP = Médio Teles Pires, Mato Grosso, Brasil.

Tabela 2. Valores de Similaridade do Coeficiente de Jaccard entre as 15 áreas e as distâncias entre cada par de localidades. Na diagonal superior da tabela seguem as distâncias em quilômetros. Na diagonal inferior da tabela seguem os valores do Coeficiente de Similaridade de Jaccard entre cada par de localidades.

\begin{tabular}{|c|c|c|c|c|c|c|c|c|c|c|c|c|c|c|c|}
\hline & IWO & PAR & ARA & FLAM & PNMT & BAR & PNJ & MAN & XIN & PNA & PNSD & BAL & MNU & CUZ & MTP \\
\hline IWO & - & 663 & 703 & 949 & 585 & 838 & 895 & 945 & 1261 & 1057 & 2221 & 2191 & 2351 & 2280 & 1836 \\
\hline PAR & 0,5631 & - & 140 & 510 & 395 & 1372 & 1345 & 1218 & 989 & 1073 & 2728 & 2647 & 2787 & 2661 & 1825 \\
\hline ARA & 0,4905 & 0,5625 & - & 368 & 301 & 1336 & 1295 & 1132 & 847 & 954 & 2673 & 2582 & 2717 & 2580 & 1691 \\
\hline FLAM & 0,3333 & 0,3943 & 0,4117 & - & 370 & 1377 & 1283 & 1029 & 495 & 731 & 2611 & 2488 & 2598 & 2435 & 1383 \\
\hline PNMT & 0,36 & 0,5147 & 0,4925 & 0,5416 & - & 1066 & 1004 & 826 & 706 & 684 & 2378 & 2281 & 2416 & 2277 & 1455 \\
\hline BAR & 0,32 & 0,4142 & 0,4117 & 0,4285 & 0,423 & - & 201 & 532 & 1383 & 937 & 1384 & 1353 & 1521 & 1465 & 1488 \\
\hline PNJ & 0,28 & 0,338 & 0,3333 & 0,3469 & 0,3207 & 0,4042 & - & 344 & 1227 & 764 & 1383 & 1312 & 1465 & 1377 & 1285 \\
\hline MAN & 0,4653 & 0,5131 & 0,4933 & 0,4333 & 0,4062 & 0,4576 & 0,3666 & - & 902 & 425 & 1582 & 1462 & 1586 & 1448 & 985 \\
\hline XIN & 0,4117 & 0,5 & 0,4794 & 0,3898 & 0,5087 & 0,4137 & 0,3928 & 0,4848 & - & 481 & 2411 & 2247 & 2328 & 2131 & 915 \\
\hline PNA & 0,466 & 0,5526 & 0,5131 & 0,4127 & 0,5 & 0,4127 & 0,3934 & 0,5671 & 0,5303 & - & 1944 & 1793 & 1889 & 1712 & 795 \\
\hline PNSD & 0,4056 & 0,45 & 0,4675 & 0,4745 & 0,4 & 0,4032 & 0,3606 & 0,4507 & 0,523 & 0,4722 & - & 249 & 387 & 603 & 1914 \\
\hline BAL & 0,4434 & 0,4634 & 0,4625 & 0,4 & 0,397 & 0,4 & 0,3809 & 0,5507 & 0,4927 & 0,4864 & 0,6363 & - & 195 & 357 & 1696 \\
\hline MNU & 0,4054 & 0,4642 & 0,4634 & 0,4029 & 0,4411 & 0,4242 & 0,3043 & 0,4666 & 0,4929 & 0,5066 & 0,6567 & 0,6666 & - & 263 & 1706 \\
\hline CUZ & 0,3333 & 0,4266 & 0,4246 & 0,4717 & 0,4137 & 0,4181 & 0,3703 & 0,4920 & 0,4754 & 0,5156 & 0,6379 & 0,6229 & 0,6451 & - & 1459 \\
\hline MTP & 0,3131 & 0,3857 & 0,4242 & 0,4468 & 0,44 & 0,3877 & 0,3333 & 0,3333 & 0,4285 & 0,4032 & 0,3492 & 0,3283 & 0,3333 & 0,3818 & - \\
\hline
\end{tabular}




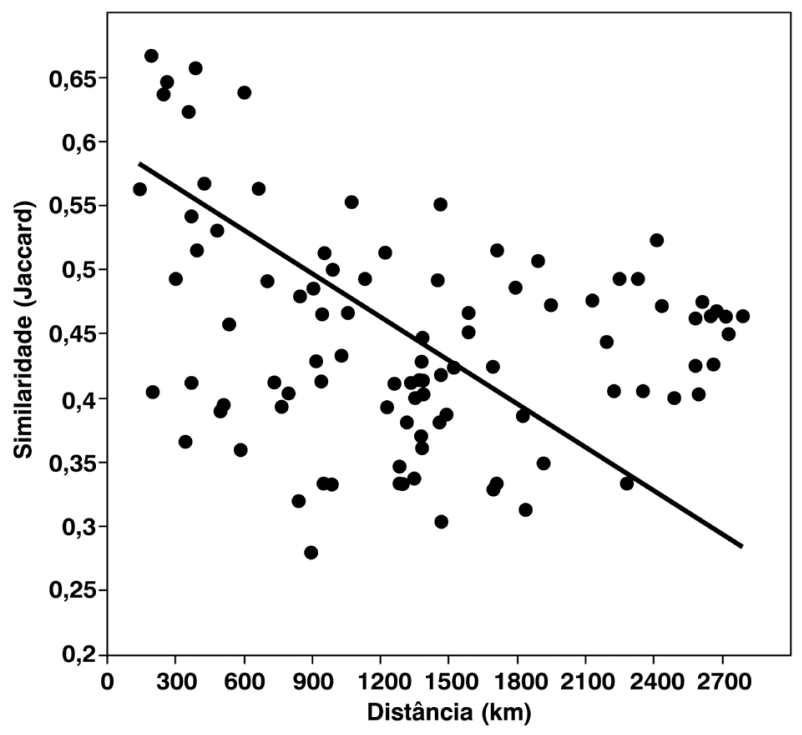

Figura 3. Correlação (correlação negativa e regressão) entre a similaridade (Jaccard) das 15 assembleias de morcegos amostradas na Amazônia e as respectivas distâncias geográficas $(\mathrm{km})$ entre essas mesmas áreas.

\section{DISCUSSÃO}

A riqueza de espécies amostrada representa $22,6 \%$ das espécies registradas para a Amazônia (Bernard et al. 2011; Paglia et al. 2012), 18\% daquelas registradas para o Brasil (Paglia et al. 2012) e 13,2\% das registradas para a América do Sul (Gardner 2008). A riqueza amostrada no presente trabalho foi similar às encontradas no PNJ $(S=31)$, em BAR $(S=35)$, na FLAM ( $=35)$ e PNMT $(S=39)$ e menor que nas demais áreas inclusas na presente análise (Anexo 1). Entretanto sabe-se que maiores esforços normalmente refletem-se em maiores riquezas (Stevens 2013). De fato, as áreas com maior riqueza de espécies foram aquelas com maiores esforços de amostragem (e.g. Voss e Emmons 1996; Simmons e Voss 1998).

$\mathrm{O}$ esforço amostral empregado no presente estudo permitiu amostrar aproximadamente $70 \%$ das espécies estimadas. Esta estimativa está abaixo daquela apontada por Simmons e Voss (1998), que sugerem que para uma área ser considerada suficientemente amostrada, $90 \%$ de sua riqueza estimada deve ser registrada. No que se refere ao número de capturas, este também ficou distante das 6.000 capturas sugeridas por Sampaio et al. (2003) para a boa amostragem de uma área na Amazônia. Dentro dessas perspectivas, poucas localidades podem atualmente ser consideradas amostradas suficientemente, o que demonstra a necessidades de novos e contínuos inventários na Amazônia.

Voss e Emmons (1996) estimaram que entre 90 e 110 espécies de morcegos podem coexistir em sintopia na Amazônia. Embora tenham sido registradas apenas 33 espécies neste estudo, é importante amostrar-se regiôes pouco conhecidas mesmo que com esforços menores (e.g. Martins et al. 2006; Moratelli et al. 2010) para que se possa aumentar o conhecimento sobre a quiropterofauna nestes locais e direcionar esforços conservacionistas, especialmente em áreas mal amostradas (Bernard et al. 2010; 2011; Martins et al. 2011).

Carollia perspicillata, assim como no presente trabalho, foi a espécie mais abundante na maioria das assembleias de morcegos estudadas na Amazônia (e.g. Reis e Peracchi 1987; Simmons e Voss 1998; Bernard 2001). Rhinophylla pumilio, Phyllostomus hastatus e Pteronotus parnellii também constam entre as espécies mais abundantes em várias localidades estudadas da Amazônia (e.g. Simmons e Voss 1998; Sampaio et al. 2003). Essas são espécies consideradas generalistas quanto ao hábitat em que vivem e também em relação aos hábitos alimentares (exceto P. parnellii que é insetívoro) (Herd 1983; Cloutier e Thomas 1992; Santos et al. 2003).

Dentre as espécies raras a maior parte correspondeu à subfamília Phyllostominae, que são reconhecidamente pouco capturáveis (Simmons e Voss 1998), e às vezes são consideradas bioindicadoras de qualidade ambiental (Fenton et al. 1992). Porém, muitas vezes espécies consideradas raras podem ser apenas pouco capturáveis (Velazco et al. 2011). Além disso, espécies raras (ou pouco capturáveis) não são as melhores opçôes quando trata-se de bioindicadores (Jones et al. 2009). De qualquer forma assembléias de morcegos monitoradas a médio e longo prazo podem funcionar como indicadores de qualidade ambiental (e.g. Willig et al. 2007; Klingbeil e Willig 2009; Jones et al. 2009). Porém, este não é um padrão geral e esse papel bioindicador deve ser visto com restriçóes (Cunto e Bernard 2012). Entre as ocorrências mais importantes destaca-se Phyllostomus latifolius que encontra atualmente no MTP seu limite sul de distribuição, sendo possível que essa espécie ocorra em toda a Amazônia (Miranda e Rubio 2011) e náo apenas na porção norte da Amazônia como se acreditava anteriormente (e.g. Gardner 2008). Outras duas espécies de interesse são: Trinycteris nicefori e Platyrrhinus fusciventris, cujas distribuiçóes foram ampliadas com o presente estudo em aproximados $500 \mathrm{~km}$ para T. nicefori $1000 \mathrm{~km}$ para P. fusciventris e atualmente encontram no MTP seu limite sul de distribuição (Gardner 2008; Velazco et al. 2010). Além dessas, o presente registro de T. cirrhosus é o segundo para o estado do Mato Grosso, sendo o primeiro registro no bioma Amazônia no estado (Souza et al. 2013).

Entre os grupos funcionais presentes no MTP, os morcegos frugívoros foram mais representados tanto em número de espécies quanto em número de indivíduos capturados. Esse padrão também foi registrado em outras comunidades de morcegos da Amazônia (Reis e Peracchi 1987; Bernard 2001) e possivelmente reflete a seletividade do método mais comumente empregado (redes de neblina armadas no subbosque) (Simmons e Voss 1998). Nos fragmentos florestais do BDFFP em Manaus, Sampaio et al. (2003) encontraram equivalência entre os Insetívoros aéreos, os Catadores 
animalívoros e os Frugívoros com 19 espécies em cada grupo funcional. Insetívoros aéreos também foram bem representados no MTP, mesmo que possivelmente sub-amostrados pelas redes em sub-bosque. Os Insetívoros aéreos podem representar o maior grupo funcional em várias localidades, especialmente em áreas onde houve esforço de longo prazo e também onde foram feitos esforços com redes de neblina armadas no dossel da floresta (Simmons e Voss 1998). Os Nectarívoros, Onívoros e Hematófagos também tiveram suas riquezas de acordo com o que já foi registrado em outras localidades amazônicas (e.g. Reis e Peracchi 1987; Simmons e Voss 1998; Bernard 2001).

A correlação negativa entre similaridades e distâncias, embora significativa, pode explicar apenas $6 \%$ da similaridade de morcegos entre as áreas amostradas. De fato, na Análise de Agrupamento percebe-se agrupamentos que parecem responder às correlaçóes espaciais: Amazônia Ocidental (BAL, MNU, PNSD e CUZ), Amazônia Central (MAN, PNA e XIN), Escudo Guiano (PAR, ARA e IWO) e as localidades do estado do Amapá (FLAM e PNMT). Por outro lado, a assembleia de morcegos do MTP náo se agrupa às localidades mais próximas e seu baixo suporte da Análise de Agrupamento também reflete sua dissimilaridade com outras áreas. $\mathrm{O}$ ramo onde se agrupam BAR, MTP, PNMT e FLAM parece não responder à proximidade entre áreas mas sim a um agrupamento "metodológico", sendo que essas áreas foram amostradas por menores esforços de campo e registrando menores riquezas (Martins et al. 2006; Moratelli et al. 2010; presente trabalho). Os agrupamentos também podem e devem estar refletindo outros fatores que não a proximidade geográfica, como fatores abióticos (temperatura, umidade, etc.) ou fatores biológicos (coocorrências, competição, fitofisionomia, etc.) não abordados na presente análise. Além disso, também é possível que a diferença (ou pouca similaridade) entre a assembleia do MTP e da outras localidades amostradas possa também refletir o relativo "isolamento" do MTP em relação às demais áreas, sendo o MTP a única área amostrada na regiāo sul da Amazônia.

\section{CONCLUSÃO}

O presente estudo registrou 33 espécies de morcegos pretencentes a seis famílias com um esforço de campo que amostrou cerca de $71 \%$ da riqueza estimada. A importância desse trabalho é destacada por ser o primeiro estudo de morcegos para a região sul da Amazônia. Além disso, por se tratar de uma assembleia diferenciada dentro do contexto regionalizado da Amazônia, reforça-se a importância de estudos nessa região, além de imcrementar os esforços de conservação no sul da Amazônia . Outros estudos poderão revelar como essa grande quantidade de espécies partilham o mesmo ambiente. Sugere-se que em futuras amostragens incluam-se redes de neblina no dossel e sub-dossel da floresta, bem como buscas por abrigos e detectores de ultra-som.

\section{AGRADECIMENTOS}

Agradecemos ao Conselho Nacional de Desenvolvimento Tecnológico e Científico (CNPq) pelas bolsas de estudos de Marcelo B. G. Rubio e Fernando Carvalho. Agradecemos à Coordenação de Aperfeiçoamento de Pessoal de Nível Superior (CAPES) pelas bolsas de estudos de Luciana Zago e Itiberê P. Bernardi. Agradecemos também aos Biólogos Raphael E. F. Santos, Sérgio Lopes Oliveira e Rafael Luchesi Balestrin pelo apoio logístico durante as campanhas de campo.

\section{BIBLIOGRAFIA CITADA}

Barnett, A.A.; Sampaio, E.M.; Kalko, E.K.V.; Shapley, R.L.; Fischer, E.; Camargo, G.; Rodríguez-Herrera, B. 2006. Bats of Jaú National Park, Central Amazônia, Brazil. Acta Chiropterologica, 8: 103-128.

Bernard, E. 2001. Species list of bats (Mammalia, Chiroptera) of Santarém area, Pará State, Brazil. Revista Brasileira de Zoologia, 18: 455-463.

Bernard, E.; Aguiar, L.M.S.; Machado, R.B. 2010. Discovering the Brazilian bat fauna: a task for two centuries? Mammal Review, 2010: 1-17.

Bernard, E.; Tavares, V.; Sampaio, E. 2011. Compilação atualizada das espécies de morcegos (Chiroptera) para a Amazônia Brasileira. Biota Neotropica, 11: 1-12.

Bianconi, G.V.; Straube, F.C. 2002. Sobre a grandeza e a unidade utilizada para estimar o esforço de captura com utilização de redes-de-neblina. Chiroptera Neotropical, 8: 150-152.

Bianconi, G.V.; Mikich, S.B.; Pedro, W.A. 2004. Diversidade de morcegos (Mammalia, Chiroptera) em remanescentes florestais do município de Fênix, noroeste do Paraná, Brasil. Revista Brasileira de Zoologia, 21: 943-954.

Bush, M.B.; Oliveira, P.E. 2006. The rise and fall of the refugial Hypothesis of Amazonian Speciation: a paleo-ecological perspective. Biota Neotropica, 6: 1-20.

Carvalho, F.; Fabián, M.E.; Menegheti, J.O. 2013. Vertical structure of an assemblage of bats (Mammalia: Chiroptera) in a fragment of Atlantic Forest in Southern Brazil. Zoologia, 30: 491-498.

Cloutier, D.; Thomas, D.W. 1992. Carollia perspicillata. Mammalian Species, 417: 1-9.

Cunto, G.C.; Bernard, E. 2012. Neotropical bats as indicators of enviromental disturbance: what is the emerging message? Acta Chiropterologica, 14: 143-151.

Fenton, M.B.; Acharya, L.; Audet, D.; Hickey, M.B.C.; Merriman, C.; Obrist, M.K.; Syme, D.M.; Adkins, B. 1992. Phyllostomid bats (Chiroptera: Phyllostomidae) as indicators of habitat disruption in the neotropics. Biotropica, 24 (3): 440-446, 1992.

Gardner, A.L. 2008. Mammals of South America. Volume 1. Marsupials, Xenarthrans, Schrews and Bats. The University of Chicago Press, Londres and Chicago, 2008, 669p.

Gualda-Barros, J.; Nascimento, F.O.; Amaral, M.K. 2012. A new species of Callicebus Thomas, 1903 (Primates, Pitheciidae) from the States of Mato Grosso and Pará, Brazil. Papéis Avulsos de Zoologia, 52: 261-279. 
Haffer, J. 1969. Speciation in Amazonian forest birds. Science, 165: 131-137.

Haffer, J.; Prance, G.T. 2002. Impulsos climáticos da evolução na Amazônia durante o Cenozóico: sobre a teoria dos Refúgios da diferenciação biótica. Estudos Avançados, 16: 175-206.

Hammer, Ø. 20I 2. PAST. Paleontological Statistic. Version 2.14. Reference Manual. Natural History Museum University of Oslo, Oslo, 2012, 225p.

Herd, R.M. 1983. Pteronotus parnellii. Mammalian species, 209; 1-5.

Hershkovitz, P. 1977. Living New World monkeys (Platyrrhini). Vol. 1. University of Chicago Press, Chicago, 1977, 1132p.

Heyer, W.R.; Coddington, J.A.; Kress, W.J.; Acevedo, P.; Cole, D.; Erwin, T.L. et al. 1999. Amazônian biotic data and conservation decisions. Ciência e Cultura, 51: 372-385.

IBGE. 2002. Mapa climático do Brasil. Instituto Brasileiro de Geografia e Estatística, Rio de Janeiro. (http://mapas.ibge.gov. br/tematicos). Acesso em: 23/04/2013.

Jones, G.; Jacobs, D.S.; Kunz, T.H.; Willig, M.R.; Racey, P.A. 2009. Carpe noctem: the importance of bats as bioindicators. Endangered Species Research, 8: 93-115.

Klingbeil, B.T.; Willig, M.R. 2009. Guild-specific responses of bats to landscape composition and configuration in fragmented Amazonian rainforest. Journal of Applied Ecology, 46: 203-2013.

Kunz, T.H.; Pierson, E.D. 1994. Bats of the world: An introduction. In: Nowak, R.M. (Ed.). Walker's Bats of the world. The Johns Hopkins University Press, Baltimore, Maryland, p. 1-46.

Lim, B.K.; Engstron, M.D. 2001. Species diversity of bats (Mammalia; Chiroptera) in Iwokrama Forest, Guyana, and the Guianan subregion: implications for conservation. Biodiversity and Conservation, 10: 613-657.

Magurran, A.E. 2011. Medindo a diversidade biológica. Editora UFPR, Curitiba, 2011, 261p.

Martins, A.C.; Bernard, E.; Gregorin, R. 2006. Inventários rápidos de morcegos (Mammlaia, Chiroptera) em três unidades de conservação do Amapá, Brasil. Revista Brasileira de Zoologia, 23: $1175-1184$.

Martins, A.C.; Bernard, E.; Gregorin, R.; Silva, W.A.S. 2011. Filling data gaps on the diversity and distribution of Amazônian bats (Chiroptera): The case of Amapá, easternmost Brazil. Zoologia, 28: 177-185.

Miranda, J.M.D.; Rubio, M.B.G. 2011. Ampliação da distribuição de Phyllostomus latifolius (Thomas, 1901) para o sul da Amazônia, Mato Grosso, Brasil (Chiroptera: Phyllostomidae). Chiroptera Neotropical, 17: 1013-1016.

Moratelli, R.; Dias, D.; Bonvicino, C.R. 2010. Estrutura e análise zoogeográfica de uma taxocenose de morcegos no norte do Estado do Amazonas, Brasil. Chiroptera Neotropical, 16: 661-671.

Nogueira, M.R.; Pol, A.; Peracchi, A.L. 1999. New records of bats from Brazil with a list of additional species for the chiropteran fauna of the state of Acre, western Amazon basin. Mammalia, 63: 363-368.

Paglia, A.P.; Fonseca, G.A.B.; Rylands, A. B.; Herrmann, G.; Aguiar, L. M. S.; Chiarello, A. G. et al. 2012. Lista anotada dos mamíferos do Brasil - 2a Edição. Occasional Papers in Conservation Biology, 6: 1-76.
Passos, F.C.; Silva, W.R.; Pedro, W.A.; Bonin, M. 2003. Frugivoria em morcegos (Mammalia, Chiroptera) no Parque Estadual Intervales, sudeste do Brasil. Revista Brasileira de Zoologia, 20: 511-517.

Reis, N.R.; Peracchi, A.L. 1987. Quirópteros da região de Manaus, Amazonas, Brasil (Mammalia, Chiroptera). Boletim do Museu Paraense Emílio Goeldi. Série Zoologia, 3: 161-182.

Reis, N.R.; Peracchi, A.L.; Pedro, W.A.; Lima, I.P. 2007. Morcegos do Brasil. Nélio R. dos Reis, Londrina, 2007, 253p.

Rosenzweig, M.L.; Sandlin, E.A. 1997. Species diversity and attitudes: listening to area's signal. Oikos, 80: 172-176.

Sampaio, E.M.; Kalko, E.K.V.; Bernard, E.; Rodríguez-Herrera, B.; Handley Jr., C.O. 2003. A biodiversity assessment of bats (Chiroptera) in a tropical lowland rainforest of Central Amazônia, including methodological and conservation considerations. Studies on Neotropical Fauna and Environment, 38: 17-31.

Santos, M.; Aguirre, L.F.; Vázquez, L.B.; Ortega, J. 2003. Pyllostomus hastatus. Mammalian Species, 722: 1-6.

Sikes, R.S.; Gannon, W.L.; The Animal Care and Use Committee of the American Society of Mammalogists. 2011. Guidelines of the American Society of Mammalogists for the use of wild mammals in research. Journal of Mammalogy, 92: 235-253.

Simmons, N.B.; Voss, R.S. 1998. The mammals of Paracou, French Guiana: a neotropical lowland rainforest fauna part 1. Bats. Bulletin of the American Museum of Natural History, 237: 1-279.

Souza, R.F.; Tenório, R.C.C.O.; Faria, K.C. 2013. First Record of Trachops cirrhosus (Spix, 1823) (Chiroptera: Phyllostomidae) for the state of Mato Grosso, Central-West region, Brazil. CheckList, 9: 1527-1529.

Stevens, R.D. 2013. Gradients of bat diversity in Atlantic Forest of South America: environmental seasonality, sampling effort and spatial autocorrelation. Biotropica, 45: 764-770.

van Roosmalen, M.G.M.; van Roosmalen, T.; Mittermeier, R.A. 2002. A taxonomic review of the titi monkeys, genus Callicebus Thomas, 1903, with the description of two new species, Callicebus bernhardi and Callicebus stephennashi, from Brazilian Amazonia. Neotropical Primates, 10: 1-52.

Vanzolini, P.E. 1992. Paleoclimas e especiação em animais da América do Sul tropical. Estudos Avançados, 6: 41-65.

Velazco, P.M.; Gardner, A.L.; Patterson, B.D. 2010. Systematic of the Platyrrhinus helleri species complex (Chiroptera; Phyllostomidae), with descriptions of two new species. Zoological Journal of the Linnean Society, 159: 786-812.

Velazco, P.M.; Pacheco, V.; Meschede, A. 2011. First occurence of the rare emballonurid bat Cyttarops alecto (Thmas, 1913) in Peruonly hard to find or truly rare? Mammalian Biology, 76: 373-376.

Voss, R.S.; Emmons, L.H. 1996. Mammalian diversity in neotropical lowland rainforests: a preliminary assessment. Bulletin of American Museum of Natural History, 230: 1-115.

Willig, M.R.; Presley, S.J.; Bloch, C.P.; Hice, C.L.; Yanoviak, S.P.; Díaz, M.M. et al. 2007. Bats of lowland Amazonia: Effects of habitat alteration on abundance. Biotropica, 39: 737-746.

Recebido em 10/02/2014

Aceito em 03/07/2014 


\section{APÊNDICE}

\section{ANEXO 1}

Espécies de morcegos registradas em sub-bosque em 15 localidades amostradas na Amazônia e utilizadas nas análises de similaridade e auto-correlação espacial de morcegos da Amazônia: IWO = Iwokrama Forest, Guiana; PAR = Paracou, Guiana Francesa; ARA = Baixo Arataye, Guiana Francesa; FLAM = Floresta Nacional do Amapá, Amapá, Brasil; PNMT
= Parque Nacional das Montanhs do Tumucumaque, Amapá, Brasil; BAR = Barcelos e Santa Isabel do Rio Negro; PNJ = Parque Nacional do Jaú, Amazônas, Brasil; MAN = Manaus, Amazônas, Brasil; XIN = Baixo Xingú, Pará, Brasil; PNA = Parque Nacional da Amazônia, Amazonas/Pará, Brasil; PNSD = Parque Nacional da Serra do Divisor, Acre, Brasil; BAL = Balta, Ucayali, Peru; MNU = Parque Nacional de Manu, Madre de Dios, Peru; CUZ = Reserva Cuzco Amazônico, Madre de Dios, Peru e MTP = Médio Teles Pires, Mato Grosso, Brasil.

\begin{tabular}{|c|c|c|c|c|c|c|c|c|c|c|c|c|c|c|c|}
\hline Espécie & IWO & PAR & ARA & FLAM & PNMT & BAR & PNJ & MAN & XIN & PNA & PNSD & BAL & MNU & CUZ & MTP \\
\hline Ametrida centurio & 1 & 1 & 1 & 0 & 0 & 1 & 1 & 1 & 0 & 1 & 0 & 0 & 0 & 0 & 0 \\
\hline Anoura caudifer & 1 & 1 & 1 & 0 & 1 & 0 & 0 & 1 & 1 & 0 & 1 & 1 & 1 & 0 & 0 \\
\hline Anoura geoffroyi & 1 & 0 & 1 & 0 & 0 & 0 & 0 & 0 & 0 & 0 & 0 & 1 & 0 & 0 & 0 \\
\hline Anoura latidens & 1 & 0 & 0 & 0 & 0 & 0 & 0 & 0 & 0 & 0 & 0 & 0 & 0 & 0 & 0 \\
\hline Artibeus amplus & 1 & 0 & 0 & 0 & 0 & 0 & 0 & 0 & 0 & 0 & 0 & 0 & 0 & 0 & 0 \\
\hline Artibeus cinereus & 1 & 1 & 0 & 1 & 1 & 1 & 1 & 1 & 1 & 1 & 0 & 1 & 1 & 1 & 0 \\
\hline Artibeus concolor & 1 & 1 & 1 & 0 & 1 & 0 & 1 & 1 & 1 & 1 & 0 & 1 & 0 & 0 & 0 \\
\hline Artibeus glaucus & 1 & 0 & 0 & 0 & 0 & 0 & 0 & 0 & 0 & 0 & 0 & 0 & 1 & 0 & 0 \\
\hline Artibeus gnomus & 1 & 1 & 1 & 0 & 1 & 1 & 0 & 0 & 1 & 0 & 0 & 0 & 1 & 0 & 1 \\
\hline Artibeus lituratus & 1 & 1 & 1 & 1 & 1 & 1 & 1 & 1 & 1 & 1 & 1 & 1 & 1 & 1 & 1 \\
\hline Artibeus obscurus & 1 & 1 & 1 & 1 & 1 & 1 & 1 & 0 & 1 & 1 & 1 & 1 & 0 & 1 & 1 \\
\hline Artibeus planirostris & 1 & 1 & 1 & 1 & 1 & 1 & 1 & 1 & 1 & 1 & 1 & 1 & 1 & 1 & 1 \\
\hline Carollia brevicauda & 1 & 0 & 1 & 1 & 1 & 0 & 1 & 1 & 0 & 1 & 1 & 1 & 1 & 1 & 0 \\
\hline Carollia castanea & 0 & 0 & 0 & 0 & 0 & 0 & 0 & 0 & 0 & 0 & 1 & 1 & 1 & 1 & 0 \\
\hline Carollia perspicillata & 1 & 1 & 1 & 1 & 1 & 1 & 1 & 1 & 1 & 1 & 1 & 1 & 1 & 1 & 1 \\
\hline Centronycteris maximiliani & 1 & 0 & 0 & 1 & 0 & 0 & 0 & 1 & 0 & 0 & 0 & 0 & 0 & 0 & 0 \\
\hline Chiroderma salvini & 0 & 0 & 0 & 0 & 0 & 0 & 0 & 0 & 0 & 0 & 0 & 0 & 0 & 1 & 0 \\
\hline Chiroderma trinitatum & 1 & 1 & 1 & 1 & 0 & 1 & 0 & 1 & 0 & 0 & 1 & 1 & 1 & 0 & 0 \\
\hline Chiroderma villosum & 1 & 1 & 1 & 0 & 1 & 0 & 0 & 0 & 1 & 1 & 1 & 1 & 1 & 1 & 0 \\
\hline Choeroniscus minor & 1 & 1 & 1 & 0 & 1 & 0 & 0 & 1 & 1 & 1 & 1 & 1 & 1 & 1 & 0 \\
\hline Chrotopterus auritus & 1 & 1 & 1 & 1 & 1 & 1 & 0 & 0 & 1 & 1 & 1 & 0 & 1 & 1 & 1 \\
\hline Cormura brevirostris & 1 & 1 & 1 & 0 & 0 & 1 & 1 & 1 & 0 & 1 & 0 & 0 & 0 & 0 & 0 \\
\hline Cynomops abrasus & 1 & 0 & 0 & 0 & 0 & 0 & 0 & 1 & 0 & 0 & 0 & 1 & 0 & 0 & 0 \\
\hline Cynomops greenhalli & 0 & 0 & 1 & 0 & 0 & 0 & 0 & 0 & 0 & 0 & 0 & 0 & 0 & 0 & 0 \\
\hline Cynomops paranus & 1 & 0 & 0 & 0 & 0 & 0 & 0 & 0 & 0 & 0 & 0 & 1 & 0 & 0 & 0 \\
\hline Cynomops planirostris & 0 & 0 & 1 & 0 & 0 & 0 & 0 & 0 & 0 & 0 & 0 & 0 & 0 & 0 & 0 \\
\hline Dermanura anderseni & 0 & 0 & 0 & 0 & 0 & 0 & 1 & 0 & 0 & 0 & 1 & 1 & 1 & 1 & 0 \\
\hline Desmodus rotundus & 1 & 1 & 1 & 1 & 1 & 1 & 1 & 1 & 1 & 1 & 1 & 1 & 1 & 1 & 1 \\
\hline Diameus youngi & 1 & 1 & 0 & 0 & 0 & 0 & 1 & 0 & 0 & 0 & 1 & 0 & 0 & 0 & 0 \\
\hline Diclidurus albus & 1 & 0 & 0 & 0 & 0 & 0 & 0 & 1 & 0 & 0 & 0 & 0 & 0 & 0 & 0 \\
\hline Diclidurus ingens & 1 & 0 & 0 & 0 & 0 & 0 & 0 & 0 & 0 & 0 & 0 & 0 & 0 & 0 & 0 \\
\hline Diclidurus isabelus & 1 & 0 & 0 & 0 & 0 & 0 & 0 & 0 & 0 & 0 & 0 & 0 & 0 & 0 & 0 \\
\hline Diclidurus scutatus & 0 & 0 & 1 & 0 & 0 & 0 & 0 & 0 & 0 & 0 & 0 & 0 & 0 & 0 & 0 \\
\hline
\end{tabular}


ANEXO 1. Continuação

\begin{tabular}{|c|c|c|c|c|c|c|c|c|c|c|c|c|c|c|c|}
\hline Espécie & IWO & PAR & ARA & FLAM & PNMT & BAR & PNJ & MAN & $\mathrm{XIN}$ & PNA & PNSD & BAL & MNU & CUZ & MTP \\
\hline Diphylla ecaudata & 0 & 0 & 0 & 0 & 0 & 0 & 0 & 0 & 1 & 0 & 1 & 0 & 1 & 0 & 0 \\
\hline Eptesicus brasiliensis & 1 & 0 & 0 & 0 & 0 & 0 & 0 & 1 & 0 & 0 & 0 & 1 & 0 & 0 & 0 \\
\hline Eptesicus chiriquinus & 1 & 1 & 0 & 0 & 0 & 0 & 0 & 0 & 0 & 0 & 0 & 0 & 0 & 0 & 0 \\
\hline Eptesicus furinalis & 0 & 1 & 0 & 0 & 0 & 0 & 0 & 0 & 0 & 0 & 0 & 1 & 0 & 0 & 0 \\
\hline Eumops auripendulus & 1 & 0 & 0 & 0 & 0 & 0 & 0 & 0 & 0 & 0 & 0 & 0 & 0 & 0 & 0 \\
\hline Eumops cf. bonariensis & 0 & 0 & 0 & 0 & 0 & 0 & 1 & 0 & 0 & 0 & 0 & 0 & 0 & 0 & 0 \\
\hline Eumops hansae & 1 & 0 & 1 & 0 & 0 & 1 & 0 & 0 & 0 & 0 & 0 & 0 & 0 & 0 & 0 \\
\hline Eumops trumbuli & 0 & 0 & 0 & 0 & 0 & 0 & 0 & 1 & 0 & 0 & 0 & 0 & 0 & 0 & 0 \\
\hline Eumops perotis & 0 & 0 & 0 & 0 & 0 & 1 & 0 & 0 & 0 & 0 & 0 & 0 & 0 & 0 & 0 \\
\hline Furipterus horrens & 0 & 0 & 0 & 0 & 0 & 0 & 0 & 0 & 1 & 0 & 0 & 0 & 1 & 0 & 0 \\
\hline Glossophaga comissarisi & 0 & 0 & 0 & 0 & 0 & 0 & 0 & 0 & 0 & 0 & 0 & 0 & 1 & 0 & 0 \\
\hline Glossophaga longirostris & 1 & 0 & 0 & 0 & 0 & 0 & 0 & 0 & 0 & 0 & 0 & 0 & 0 & 0 & 0 \\
\hline Glossophaga soricina & 1 & 1 & 1 & 1 & 0 & 1 & 1 & 1 & 1 & 1 & 1 & 1 & 1 & 1 & 1 \\
\hline Glyphonycteris sylvestris & 1 & 1 & 1 & 0 & 0 & 0 & 0 & 0 & 1 & 0 & 0 & 0 & 0 & 0 & 1 \\
\hline Glyyphonycteris daviesi & 1 & 1 & 0 & 0 & 0 & 0 & 0 & 0 & 1 & 0 & 0 & 0 & 0 & 0 & 0 \\
\hline Lampronycteris brachyotis & 1 & 0 & 0 & 0 & 0 & 0 & 0 & 0 & 0 & 1 & 0 & 0 & 0 & 0 & 1 \\
\hline Lasiurus atratus & 1 & 0 & 0 & 0 & 0 & 0 & 0 & 0 & 0 & 0 & 0 & 0 & 0 & 0 & 0 \\
\hline Lasiurus blossevillii & 1 & 1 & 0 & 0 & 0 & 0 & 0 & 1 & 0 & 0 & 0 & 1 & 0 & 0 & 0 \\
\hline Lasiurus ega & 0 & 0 & 0 & 0 & 0 & 0 & 0 & 0 & 0 & 0 & 1 & 1 & 1 & 1 & 0 \\
\hline Lichonycteris degener & 0 & 1 & 0 & 0 & 0 & 0 & 0 & 1 & 0 & 1 & 0 & 0 & 0 & 0 & 0 \\
\hline Lionycteris spurrelli & 1 & 0 & 1 & 1 & 1 & 0 & 0 & 0 & 0 & 0 & 0 & 0 & 0 & 0 & 1 \\
\hline Lonchophylla thomasi & 1 & 1 & 1 & 1 & 1 & 1 & 1 & 1 & 1 & 1 & 1 & 1 & 1 & 1 & 1 \\
\hline Lonchorrhina aurita & 1 & 0 & 0 & 0 & 0 & 0 & 0 & 0 & 0 & 1 & 0 & 0 & 0 & 0 & 0 \\
\hline Lonchorrhina marinkellei & 0 & 0 & 1 & 0 & 0 & 0 & 0 & 0 & 0 & 0 & 0 & 0 & 0 & 0 & 0 \\
\hline Lophostoma brasiliense & 1 & 1 & 0 & 0 & 1 & 0 & 0 & 0 & 1 & 1 & 0 & 1 & 1 & 0 & 1 \\
\hline Lophostoma carrikieri & 1 & 1 & 0 & 0 & 0 & 0 & 1 & 0 & 0 & 1 & 0 & 1 & 0 & 0 & 0 \\
\hline Lophostoma schulzi & 1 & 1 & 1 & 0 & 1 & 0 & 0 & 0 & 0 & 0 & 0 & 0 & 0 & 0 & 0 \\
\hline Lophostoma silvicolum & 1 & 1 & 1 & 1 & 1 & 1 & 0 & 1 & 1 & 1 & 1 & 1 & 1 & 1 & 0 \\
\hline Macrophyllum macrophyllum & 1 & 0 & 1 & 0 & 0 & 1 & 1 & 1 & 1 & 1 & 1 & 1 & 1 & 0 & 0 \\
\hline Mesophylla macconnellii & 1 & 1 & 1 & 0 & 0 & 0 & 1 & 1 & 1 & 0 & 1 & 1 & 1 & 1 & 1 \\
\hline Micronycteris brosseti & 1 & 1 & 0 & 0 & 0 & 0 & 0 & 0 & 0 & 0 & 0 & 0 & 0 & 0 & 0 \\
\hline Micronycteris hirsuta & 1 & 1 & 0 & 0 & 0 & 0 & 0 & 0 & 0 & 0 & 1 & 0 & 0 & 0 & 0 \\
\hline Micronycteris homezi & 1 & 0 & 0 & 0 & 0 & 0 & 0 & 0 & 0 & 0 & 0 & 0 & 0 & 0 & 0 \\
\hline Micronycteris megalotis & 1 & 1 & 1 & 0 & 0 & 0 & 0 & 1 & 1 & 1 & 1 & 1 & 1 & 1 & 0 \\
\hline Micronycteris microtis & 1 & 1 & 0 & 1 & 1 & 0 & 0 & 0 & 0 & 0 & 0 & 0 & 0 & 0 & 0 \\
\hline Micronycteris minuta & 1 & 1 & 0 & 1 & 0 & 0 & 0 & 0 & 0 & 1 & 1 & 0 & 1 & 1 & 0 \\
\hline Micronycteris schmidtorum & 0 & 1 & 0 & 0 & 1 & 0 & 0 & 0 & 0 & 1 & 0 & 0 & 1 & 0 & 0 \\
\hline Mimon bennettii & 1 & 1 & 1 & 1 & 0 & 0 & 0 & 0 & 0 & 0 & 0 & 0 & 0 & 0 & 0 \\
\hline Mimon crenulatum & 1 & 1 & 1 & 1 & 1 & 1 & 0 & 1 & 0 & 1 & 0 & 1 & 1 & 1 & 0 \\
\hline Molossops neglectus & 1 & 0 & 0 & 0 & 0 & 0 & 0 & 0 & 0 & 0 & 0 & 0 & 0 & 0 & 0 \\
\hline
\end{tabular}


ANEXO 1. Continuação

\begin{tabular}{|c|c|c|c|c|c|c|c|c|c|c|c|c|c|c|c|}
\hline Espécie & IWO & PAR & ARA & FLAM & PNMT & BAR & PNJ & MAN & $\mathrm{XIN}$ & PNA & PNSD & BAL & MNU & CUZ & MTP \\
\hline Molossus barnesi & 0 & 1 & 0 & 0 & 0 & 0 & 0 & 0 & 0 & 0 & 0 & 0 & 0 & 0 & 0 \\
\hline Molossus molossus & 1 & 1 & 1 & 1 & 0 & 1 & 1 & 1 & 0 & 0 & 1 & 1 & 1 & 1 & 1 \\
\hline Molossus pretiosus & 1 & 0 & 0 & 0 & 0 & 0 & 0 & 0 & 0 & 0 & 0 & 0 & 0 & 0 & 0 \\
\hline Molossus rufus & 1 & 1 & 1 & 0 & 0 & 0 & 0 & 1 & 0 & 1 & 0 & 0 & 0 & 0 & 1 \\
\hline Myotis albescens & 1 & 0 & 0 & 1 & 0 & 1 & 0 & 1 & 1 & 0 & 1 & 1 & 1 & 1 & 0 \\
\hline Myotis nigricans & 1 & 1 & 1 & 0 & 0 & 0 & 0 & 1 & 0 & 1 & 0 & 1 & 1 & 1 & 1 \\
\hline Myotis riparius & 1 & 1 & 1 & 0 & 1 & 1 & 0 & 0 & 1 & 0 & 1 & 1 & 1 & 1 & 1 \\
\hline Myotis simus & 0 & 0 & 0 & 0 & 0 & 0 & 0 & 0 & 0 & 0 & 0 & 1 & 1 & 0 & 0 \\
\hline Neoplatymops mattogrossensis & 1 & 0 & 0 & 0 & 0 & 0 & 0 & 0 & 1 & 0 & 0 & 0 & 0 & 0 & 0 \\
\hline Noctilio albiventris & 1 & 1 & 0 & 0 & 0 & 1 & 1 & 1 & 1 & 1 & 1 & 1 & 1 & 1 & 1 \\
\hline Noctilio leporinus & 1 & 1 & 0 & 0 & 1 & 1 & 0 & 1 & 0 & 0 & 0 & 0 & 1 & 0 & 0 \\
\hline Nyctinomops laticaudatus & 0 & 0 & 1 & 0 & 0 & 0 & 0 & 0 & 0 & 1 & 0 & 0 & 1 & 0 & 0 \\
\hline Nyctinomops macrotis & 1 & 0 & 0 & 0 & 0 & 0 & 0 & 0 & 0 & 0 & 0 & 0 & 0 & 0 & 0 \\
\hline Peropteryx kappleri & 0 & 1 & 0 & 0 & 0 & 0 & 0 & 0 & 0 & 0 & 0 & 0 & 0 & 0 & 0 \\
\hline Peropteryx leucoptera & 1 & 0 & 0 & 0 & 0 & 0 & 0 & 1 & 0 & 1 & 0 & 0 & 0 & 1 & 0 \\
\hline Peropteryx macrotis & 1 & 0 & 1 & 0 & 0 & 0 & 0 & 1 & 1 & 0 & 0 & 0 & 0 & 0 & 0 \\
\hline Phylloderma stenops & 1 & 1 & 1 & 0 & 1 & 1 & 0 & 0 & 0 & 1 & 0 & 0 & 1 & 0 & 1 \\
\hline Phyllostomus discolor & 1 & 1 & 1 & 1 & 1 & 0 & 0 & 1 & 1 & 1 & 1 & 0 & 0 & 0 & 1 \\
\hline Phyllostomus elongatus & 1 & 1 & 1 & 1 & 1 & 0 & 1 & 1 & 1 & 1 & 1 & 1 & 1 & 1 & 1 \\
\hline Phyllostomus hastatus & 1 & 1 & 1 & 1 & 1 & 1 & 1 & 1 & 1 & 1 & 1 & 1 & 1 & 1 & 1 \\
\hline Phyllostomus latifolius & 1 & 0 & 1 & 0 & 0 & 0 & 1 & 0 & 0 & 0 & 0 & 0 & 0 & 0 & 1 \\
\hline Platyrrhinus aurarius & 1 & 0 & 0 & 0 & 0 & 0 & 0 & 0 & 0 & 0 & 0 & 0 & 0 & 0 & 0 \\
\hline Platyrrhinus brachycephalus & 1 & 0 & 0 & 0 & 0 & 0 & 0 & 0 & 0 & 0 & 1 & 1 & 1 & 0 & 0 \\
\hline Platyrrhinus cf. helleri & 1 & 1 & 1 & 1 & 1 & 1 & 0 & 1 & 1 & 1 & 1 & 1 & 1 & 1 & 1 \\
\hline Platyrrhinus infuscus & 0 & 0 & 0 & 0 & 0 & 0 & 0 & 0 & 0 & 0 & 1 & 1 & 1 & 1 & 0 \\
\hline Platyrrhinus lineatus & 0 & 0 & 1 & 0 & 1 & 0 & 0 & 0 & 0 & 0 & 0 & 0 & 0 & 0 & 0 \\
\hline Promops centralis & 1 & 0 & 0 & 0 & 0 & 0 & 0 & 0 & 0 & 0 & 1 & 0 & 0 & 0 & 0 \\
\hline Pteronotus gymnonotus & 1 & 0 & 0 & 0 & 0 & 0 & 0 & 0 & 0 & 0 & 0 & 0 & 0 & 0 & 0 \\
\hline Pteronotus parnellii & 1 & 1 & 1 & 1 & 1 & 0 & 0 & 1 & 1 & 1 & 0 & 0 & 0 & 0 & 1 \\
\hline Pteronotus personatus & 1 & 0 & 0 & 0 & 0 & 0 & 0 & 0 & 0 & 1 & 0 & 0 & 0 & 0 & 1 \\
\hline Rhinophylla fisherae & 0 & 0 & 0 & 0 & 0 & 0 & 0 & 0 & 1 & 1 & 1 & 0 & 0 & 0 & 0 \\
\hline Rhinophylla pumillio & 1 & 1 & 1 & 1 & 1 & 1 & 1 & 1 & 1 & 1 & 1 & 1 & 1 & 1 & 1 \\
\hline Rhogeessa io & 0 & 0 & 0 & 0 & 0 & 1 & 0 & 0 & 0 & 0 & 0 & 0 & 0 & 0 & 0 \\
\hline Rhynchonycteris naso & 1 & 0 & 0 & 1 & 1 & 1 & 1 & 1 & 1 & 1 & 1 & 1 & 1 & 1 & 1 \\
\hline Saccopteryx canescens & 1 & 0 & 0 & 0 & 0 & 0 & 1 & 0 & 1 & 1 & 0 & 0 & 0 & 0 & 0 \\
\hline Saccopteryx gymnura & 1 & 1 & 0 & 0 & 0 & 0 & 0 & 0 & 0 & 0 & 0 & 0 & 0 & 0 & 0 \\
\hline Saccopteryx leptura & 1 & 1 & 1 & 0 & 0 & 1 & 0 & 1 & 1 & 1 & 1 & 1 & 1 & 1 & 0 \\
\hline Saccopetryx bilineata & 1 & 1 & 1 & 0 & 1 & 1 & 1 & 1 & 1 & 1 & 1 & 1 & 1 & 1 & 0 \\
\hline Sphaeronycteris toxophyllum & 0 & 0 & 0 & 0 & 0 & 0 & 0 & 0 & 0 & 0 & 0 & 0 & 1 & 0 & 0 \\
\hline Sturnira lilium & 1 & 1 & 1 & 0 & 0 & 0 & 0 & 1 & 1 & 1 & 1 & 1 & 1 & 1 & 0 \\
\hline
\end{tabular}


ANEX0 1. Continuação

\begin{tabular}{|c|c|c|c|c|c|c|c|c|c|c|c|c|c|c|c|}
\hline Espécie & IWO & PAR & ARA & FLAM & PNMT & BAR & PNJ & MAN & XIN & PNA & PNSD & BAL & MNU & CUZ & MTP \\
\hline Sturnira magna & 0 & 0 & 0 & 0 & 0 & 0 & 0 & 0 & 0 & 0 & 1 & 0 & 0 & 0 & 0 \\
\hline Sturnira tildae & 1 & 1 & 1 & 1 & 1 & 0 & 1 & 0 & 1 & 1 & 1 & 1 & 1 & 1 & 0 \\
\hline Tonatia saurophila & 1 & 1 & 1 & 1 & 1 & 1 & 1 & 1 & 1 & 1 & 1 & 1 & 1 & 0 & 0 \\
\hline Trachops cirrhosus & 1 & 1 & 1 & 1 & 1 & 1 & 1 & 1 & 1 & 1 & 1 & 1 & 1 & 1 & 1 \\
\hline Trinycteris nicefori & 1 & 1 & 1 & 1 & 1 & 1 & 0 & 1 & 1 & 1 & 1 & 1 & 0 & 0 & 1 \\
\hline Tyroptera tricolor & 1 & 1 & 1 & 0 & 0 & 1 & 0 & 1 & 0 & 1 & 1 & 1 & 1 & 1 & 0 \\
\hline Uroderma bilobatum & 1 & 1 & 1 & 1 & 1 & 0 & 0 & 1 & 1 & 1 & 1 & 1 & 1 & 1 & 1 \\
\hline Uroderma magnirostrum & 0 & 0 & 0 & 0 & 0 & 0 & 0 & 1 & 1 & 1 & 0 & 1 & 1 & 1 & 0 \\
\hline Vampyressa melissa & 0 & 0 & 1 & 0 & 0 & 0 & 0 & 0 & 0 & 0 & 0 & 0 & 0 & 0 & 0 \\
\hline Vampyressa thyone & 0 & 0 & 1 & 1 & 0 & 0 & 0 & 0 & 0 & 0 & 1 & 1 & 1 & 1 & 1 \\
\hline Vampyriscus bidens & 1 & 0 & 1 & 0 & 0 & 0 & 0 & 0 & 0 & 1 & 1 & 1 & 1 & 0 & 0 \\
\hline Vampyriscus brocki & 1 & 1 & 1 & 0 & 0 & 0 & 1 & 0 & 1 & 0 & 0 & 0 & 0 & 0 & 0 \\
\hline Vampyrodes caraccioli & 1 & 0 & 0 & 1 & 0 & 0 & 0 & 0 & 0 & 0 & 1 & 1 & 1 & 0 & 0 \\
\hline Vampyrum spectrum & 1 & 1 & 1 & 1 & 1 & 0 & 0 & 1 & 0 & 1 & 1 & 0 & 1 & 1 & 0 \\
\hline Riqueza (S) & 97 & 64 & 61 & 35 & 39 & 35 & 31 & 51 & 47 & 54 & 52 & 56 & 59 & 43 & 33 \\
\hline
\end{tabular}

